treatment was extracted from digital health records. Patients sutured under GA were reviewed for contra-indications to Ketamine, determining the number potentially suitable for procedural sedation in retrospect. A literature search was used to identify healthcare costs of laceration closure under GA, versus the same procedure under Ketamine sedation. Admission cost per patient was gained by communication with relevant ward managers. Retrospective economic savings were calculated to ascertain the theoretical annual savings of introducing a Ketamine sedation list in the PED.

Results 234 patients were referred to OMFS for facial lacerations. $162(69.2 \%)$ of these were deemed to require closure with sutures. 107 were able to tolerate closure under local anaesthetic while 55 required admission and suturing under GA. Of the children sutured under GA, 47 (85.5\%) were deemed to be retrospectively suitable for Ketamine sedation and the remaining $8(14.5 \%)$ had contra-indications. The cost saving of using Ketamine sedation over GA is $£ 69$ per patient and the admission cost per patient per day is $£ 306$. The retrospective theoretical annual healthcare saving was approximately $£ 18,543$.

Conclusion There is scope for introduction of a procedural Ketamine sedation list for paediatric facial lacerations that are currently being sutured under GA. This would reduce healthcare costs dramatically as well as optimising resources in the PED.

\section{G244(P) TREATMENT OF FEVER IN CHILDREN WITH SICKLE CELL DISEASE}

'S Ravindran, ${ }^{2} S$ Chakravorty, 'R Mitchell. 'Paediatric Emergency Department, King's College Hospital NHS FT, London, UK; ${ }^{2}$ Department of Paediatric Haematology, King's College Hospital NHS FT, London, UK

10.1136/archdischild-2020-rcpch.211

Aims Fever is an important sign in children with sickle cell disease (SCD), often being the only indicator of serious and potentially life-threatening secondary bacterial infection. Treatment of febrile children with SCD in our Paediatric Emergency Department (PED) can be variable, including escalation of care of those who are unwell and pragmatic management of those who are well. We therefore audited the management of fever with SCD presenting to PED and developed guidance to aid management, particularly looking at whether ambulatory nursing teams can be utilised to manage 'low-risk' patients.

Methods We analysed the management of SCD children presenting to PED with fever over a one year period. Using these results, we developed guidance for febrile children with SCD, based on risk stratification.

Results 28 children with SCD presented between July 2018 July 2019, of whom 22 were admitted and 6 discharged from PED. Diagnoses among these patients are displayed in table 1.

Antibiotic treatment was consistent in the management of fever due to acute chest syndrome, but was otherwise variable. One third of children discharged (2/6) received oral antibiotic treatment to complete at home. None of the discharged children were referred to the ambulatory nursing team for review. Subsequently we developed guidance to standardise clinical assessment, direct antibiotic choice, give clear risk-stratification criteria and advice on management of low-risk fevers of SCD children in the community.
Abstract G244(P) Table 1 Diagnoses among febrile children with SCD presenting to PED

\begin{tabular}{lll}
\hline Outcome & Diagnosis & Number \\
\hline Admitted: & Acute chest syndrome & 11 \\
& Vaso-occlusive crisis: abdominal & 7 \\
& Vaso-occlusive crisis: limb & 2 \\
& Fever without obvious source & 1 \\
& Influenza & 1 \\
& Upper respiratory tract infection & 2 \\
Discharged: & Vaso-occlusive crisis: limb & 3 \\
& Peri-orbital cellulitis & 1 \\
& & Total $=28$ \\
\hline
\end{tabular}

Conclusions Fever may indicate serious bacterial infection in children with SCD, requiring prompt and specific antibiotic treatment. Implementation of a guideline will help tailor treatment to the most likely cause of fever and risk-stratify these children accordingly. Utilisation of ambulatory nursing teams should be encouraged to promote admission avoidance and ensure low-risk children can be managed safely at home.

\section{G245(P) ESTIMATING WEIGHT IN PAEDIATRIC TRAUMA PATIENTS - AN ONGOING CHALLENGE WITH A NEW SOLUTION}

G Campbell, S Foster, M McGlone. Emergency Department, NHS Greater Glasgow and Clyde, Glasgow, UK

10.1136/archdischild-2020-rcpch.212

The administration of drugs and fluid, most of which are given as the dose per $\mathrm{kg}$ of body weight, is a vital component in the management of the seriously injured child. Obtaining an accurate weight on arrival may not be immediately achievable because of instability, immobilisation and ongoing resuscitation.

The paediatric STAG (Scottish trauma audit group) data from * hospital was used to identify patients over 12 months. Clinical notes were reviewed to assess if weight was estimated or accurately measured.

56 paediatric trauma patients who met the STAG inclusion criteria presented to * over the 12 month period.

27/56 (48\%) patients had an estimated weight on arrival; 5 were estimated using the 'old' APLS formula, 12 using the 'new' formulae and for the remaining 10 the method used was unclear.

Of those who initially had an estimated weight only $7 / 27$ (26\%) went on to have an accurate weight obtained at any time during their admission. In 5 patients, weight was underestimated (mean -12\%) and in 2 patients, weight was overestimated (mean +19\%). The weight estimation error ranged from negative $28 \%$ to positive $20 \%$. Assessing the percentage error of estimated weight was challenging in this group of patients due to the small number on which an accurate weight was measured during this admission. Poor documentation may have been a contributing factor in some cases with the weight on a patient's drug kardex only being clearly identified as being an 'estimated' value in $66 \%$ of patients. 
There are many implications of using an estimated weight, including errors in drug doses, fluid bolus, maintenance fluids, ventilator settings and assessment of urine output. In this cohort the estimated weight that followed them throughout their admission has implications on their immediate resuscitation and extended inpatient care and highlights the importance of obtaining an accurate weight at the time of arrival.

What can we do differently? We have implemented the use of a pat-slide weighing scale by MARSDEN to get an accurate weight from time of arrival. This device enables a rapid weight to be obtained during patient transfer to the trolley.

\section{G246(P) 'SEE IT, EAT IT, A\&E IT' - ACCIDENTAL POISONINGS IN A PAEDIATRIC EMERGENCY DEPARTMENT}

${ }^{1}$ A Norton, ${ }^{1} T$ Malik, ${ }^{2} \mathrm{M}$ Naravi. 'School of Medicine, University of Leeds, Leeds, UK; ${ }^{2}$ Emergency Department, Bradford Royal Infirmary, Bradford, UK

\subsection{6/archdischild-2020-rcpch.213}

Aims Many common household items, which may not be perceived as 'poisons', can have serious consequences if ingested by a curious toddler. Between 2012/13 - 2016/17, 25,591 children under five were hospitalised for accidental poisoning in England. Unfortunately, recent UK data is scarce and focuses mainly on fatal poisonings. This study aimed to understand the aetiology of all accidental poisonings presenting to a single Emergency Department (ED), in order to guide preventative advice.

Methods Electronic patient records were searched for children under 16 attending a paediatric ED with a coded diagnosis of 'Accidental Poisoning' over an eight-month period. Sixty four cases were identified for analysis.

Results Most cases (84\%) occurred in children under five but accidental poisoning was most prevalent in children aged one year (34\%). Medications were responsible for half of all poisonings (51\%) and household products responsible for 33\%. Medication was usually intended for the child themselves $(31 \%)$, the child's parents $(22 \%)$ or grandparents $(22 \%)$. Two children had previous admissions for accidental ingestion in the four months prior to the study. Children were generally clinically well. Thirty percent were discharged directly from the ED and the average length of hospital stay was 8 hours 18 minutes. Only one child was hospitalised for over 24 hours. Four children (6\%) required active treatment for poisoning. Safeguarding concerns were documented in $6 \%$ of cases and referrals to Health Visitors were suggested in $39 \%$. Families were given advice on preventing poisoning in $44 \%$ of cases.

Conclusions Accidental poisoning is a common cause of paediatric ED attendance, but cases are generally mild, requiring only a period of observation and limited investigations. Children presenting with accidental poisoning are at risk of further poisoning, yet strategies within the ED to prevent future incidents are variably applied. The young age of children suggests that unsafe storage plays an important role in the causation of accidental poisoning, so preventative strategies should focus on educating families, including grandparents, on safe storage of medications and other poisons. Health Visitors should be involved in the assessment of the home environment and an information leaflet should be distributed to all families.

\section{G247(P) VENTILATOR ASSOCIATED PNEUMONIA- ARE WE (UNDER) ESTIMATING?}

${ }^{1} \mathrm{P}$ Khemka, ${ }^{1} \mathrm{R}$ Thomas, ${ }^{2} \mathrm{~S}$ Thompson. 'Pediatric Critical Care Unit, Sheffield Children's Hospital, Sheffield, UK; ${ }^{2}$ Microbiology, Sheffield Children's Hospital, Sheffield, UK

\subsection{6/archdischild-2020-rcpch.214}

Background and Aims Ventilator associated pneumonia (VAP) is the second most common healthcare associated infection and is associated with substantial morbidity, mortality, increased hospital stay and cost of healthcare. Despite this, VAP is often under recognized and under reported and there is a lack of data on VAP in UK children. We aimed to determine VAP rates in a tertiary Pediatric Intensive Care unit by tracing positive microbiological specimens for invasively ventilated patients.

Methods This was a retrospective observational study. All patients who were admitted to the pediatric critical care unit between $01 / 01 / 2017$ to $31 / 12 / 2017$ and invasively ventilated for more than $48 \mathrm{hrs}$ were included. All positive respiratory tract specimens for the study period were identified and followed by a review of relevant case notes, nursing charts and investigations. Denominator data was collected from the hospital database. VAP was diagnosed using a combination of clinical, radiological and microbiological criteria adapted and simplified from Centers for Disease Control pneumonia flowcharts for pediatric VAP diagnosis.

Results There were 8 microbiologically confirmed cases of VAP, and 2066 ventilator-days, giving a VAP rate of 3.8/1000 ventilator days. The positive microbiological specimen was endotracheal aspirate in all the cases. Few BALs had been performed and none were diagnostic of VAP. The causative agents in all these cases were bacterial; no fungal or viral pathogen was identified. Gram negative bacteria were identified in 7 out of 8 cases (87.5\%), Escherichia coli being most common (3 cases), followed by Haemophilus influenzae (2), Pseudomonas aeruginosa (2) and Staphylococcus aureus (1 case). Meropenem or piperacillin-tazobactam were used for treatment in nearly all cases (7/8). VAP was diagnosed and recorded contemporaneously in only 1 of 8 patients.

Conclusion This study defined the VAP incidence in our institution. VAP cases without microbiological confirmation were not detected and the incidence is therefore likely to be an underestimate. Prospective surveillance using clinical criteria is planned. VAP cases were associated with very broad spectrum antibiotic use in our population. Surveillance for, and interventions to prevent, VAP will be beneficial from an antimicrobial stewardship perspective.

\section{G248(P) INTRODUCING LIVE WAITING TIMES ONLINE AS A SERVICE IMPROVEMENT FOR THE PAEDIATRIC EMERGENCY DEPARTMENT (PED)}

${ }^{1} V$ Menon, ${ }^{2} \mathrm{C}$ Wong. ${ }^{1}$ Foundation Doctor, Queen Elizabeth Hospital, University Hospitals Birmingham, Birmingham, UK; ${ }^{2}$ Paediatric Emergency Department, Warrington and Halton Hospitals NHS FT, Warrington, UK

\subsection{6/archdischild-2020-rcpch.215}

Aims Waiting times in Emergency Departments (ED) is a national problem. Using advancements in technology current live ED waiting times can be made available to the public, so that patients can decide whether to attend an ED or are better off seen in the community. 